\title{
Evidence of a Role for Local Complement Expression in a Murine Model of Progressive Glomerulonephritis
}

\author{
THOMAS R. WELCH, MARIE FRENZKE, AND DAVID WITTE
}

Divisions of Nephrology [T.R.W., M.F.], and Pathology [D.W.], Children's Hospital Medical Center, and Department of Pediatrics [T.R.W., M.F.] and Pathology [D.W.], University of Cincinnati Medical Center,

Cincinnati, Ohio, U.S.A.

\begin{abstract}
C57/B6 mice received intraperitoneal horse spleen apoferritin (4 mg) with lipopolysaccharide $(0.05 \mathrm{mg})$; control mice received $0.15 \mathrm{M} \mathrm{NaCl}$. Control and treated animals were killed weekly for $6 \mathrm{wk}$; blood and urine specimens were obtained, and tissue samples were secured. Treated animals showed evidence of significant chronic disease, with proteinuria, hematuria, and uremia. A mild glomerulonephritis was present at $2 \mathrm{wk}$, with significant proliferative glomerulonephritis at $4 \mathrm{wk}$, progressing to chronic disease with tubulointerstitial changes at $6 \mathrm{wk}$. Changes at each time period were uniform between animals. C3 mRNA was first detected by in situ hybridization at 3 wk. Message was restricted to proximal tubular and periglomerular epithelial cells. Presence of C3 message preceded the development of interstitial inflammation and fibrosis by $1-2 \mathrm{wk}$, and its location and intensity paralleled the evolving interstitial disease. Although extensive mesangial C3 protein deposits appeared early, there was never $\mathrm{C} 3$ message in glomeruli or infiltrating
\end{abstract}

\section{ABSTRACT}

cells. Before C3 message became apparent, two cytokines known to up-regulate $\mathrm{C} 3$ transcription in vitro, IL-1 and IL-6, were detected by immunohistochemistry. The temporal sequence in this model is consistent with our hypothesis that local synthesis and activation of $\mathrm{C} 3$ in tubular epithelium is important to the interstitial component of chronic glomerulonephritis. The process is independent of the deposition of circulating complement in the glomerulus, but may be triggered by glomerular cytokines.

(Pediatr Res 48: 200-205, 2000)
LPS, lipopolysaccharide
HSA, horse spleen apoferritin
PAS, periodic acid-Schiff
$\mathbf{H \& E}$, hematoxylin and eosin
MAC, membrane attack complex

A variety of primary glomerular diseases can progress to chronic renal failure. Common to all of these is involvement of the interstitium with peritubular inflammation, edema, fibrosis, and tubular atrophy (1). Progression to end-stage renal disease never occurs in the absence of these features, and their presence in the early stage of disease is often predictive of deterioration (2).

Despite recognition of this sequence, there is little understanding of the mechanisms by which glomerular inflammation triggers an interstitial process. Studies of human biopsy material from our laboratory have suggested a role for local expression of complement by the tubular epithelium (3-5). A limitation of this work has been the fact that such material represents a single time point in the evolution of a complex disease. Thus, it is not possible to place the presence of tubular complement component expression into a temporal context.

Received June 8, 1999; accepted January 17, 2000.

Correspondence and reprint requests: Thomas R. Welch, M.D., Children's Hospital Medical Center, Division of Pediatric Nephrology, 3333 Burnet Avenue, Cincinnati, OH 45229-3039, U.S.A.
In an effort to test further the hypothesis that tubular complement expression was a potential mediating factor in the progression of glomerulonephritis, we determined that an animal model would be necessary. The model would need to feature tubular complement expression, and involve a species with a defined complement system. It should begin with acute glomerular inflammation, with gradual progression to interstitial involvement, permitting assessment of the time at which local complement synthesis began. The studies reported here describe the features of such a model, and the evidence supporting a role for local expression of complement component C3.

\section{METHODS}

Animals. Six-week-old male C57/B6 mice were obtained from the Jackson Laboratory (Bar Harbor, ME, U.S.A.). The study was approved by the Animal Use Committee of the Children's Hospital Research Foundation.

Antisera and reagents. FITC-conjugated goat anti-murine $\mathrm{C} 3 \mathrm{c}$ (the major fragment of C3; Nordic, Capistrano Beach, CA, 
U.S.A.), rabbit anti-mouse IL- $1 \propto$ (Endogen, Cambridge, MA, U.S.A.) rabbit anti-mouse IL-6 (Genzyme, Cambridge, MA, U.S.A.), FITC-conjugated goat anti-rabbit IgG (Rockland, Gilbertsville, PA, U.S.A.), HSA (Sigma Chemical Co., St. Louis, MO, U.S.A.), and LPS (Calbiochem, San Diego, CA, U.S.A.) were obtained as indicated.

Riboprobes. The plasmid pBR322 containing a $2.4-\mathrm{kb}$ mouse cDNA insert for C3 was obtained from American Type Culture Collection (Rockville, MD, U.S.A.). A 541-bp product was generated by PCR using a 5' CAA GGC TTG CAA TAC CAT GAA GG 3' and a 5' CCT GTG TGG GGC TGT TAA ATG 3' oligonucleotide. The PCR product was then ligated into a PCR 2.1 TA cloning vector (Invitrogen, Carlsbad, CA, U.S.A.). Colonies were grown and checked by restriction enzyme digestion for orientation to obtain sense and antisense probes. DNA was isolated using Qiagen purification (Santa Clarita, CA, U.S.A.), linearized, and then transcribed using T7 polymerase, ${ }^{35} \mathrm{~S}-\mathrm{UTP}$, and a Promega (Madison, WI, U.S.A.) transcription kit. The RNA probes were passed over quick-spin columns (Boehringer Mannheim, Indianapolis, IN, U.S.A.) to remove unincorporated nucleotides before use.

Urine and blood samples. Blood urea nitrogen concentration was measured by the Children's Hospital Medical Center clinical laboratory. The assay used (Vitros 950, Johnson \& Johnson, Rochester, NY, U.S.A.) has a reported coefficient of variation of $1.6 \%$. A normal range for murine blood urea concentration of $12-22 \mathrm{mg} / \mathrm{dL}$ was established in 10 normal 6-week-old animals. Urine was analyzed for the presence of blood and protein by Multistix (Bayer Corporation, Elkhart, IN, U.S.A.), according to directions supplied by the manufacturer.

Tissue harvesting and processing. Animals were killed by $\mathrm{CO}_{2}$ narcosis. Immediately after death, the kidneys were dissected free and bisected. Tissue samples were frozen in M-1 embedding matrix (Shandon Lipshaw, Pittsburgh, PA, U.S.A.) for immunohistochemistry or treated with $10 \%$ formalin for routine histology or $4 \%$ paraformaldehyde for in situ hybridization.

Blood samples were obtained at the time of death by cardiac puncture. Before killing, animals were held in metabolic cages to obtain urine specimens.

Immunohistochemistry. Frozen tissue was cut into 10- $\mu \mathrm{m}$ sections, dried on positively charged slides, washed with PBS, and incubated at $37^{\circ} \mathrm{C}$ for $30 \mathrm{~min}$ with $10 \%$ normal goat serum. Excess serum was blotted, and the slides were incubated with a $1 / 50$ dilution of primary antibody for $1 \mathrm{~h}$ at $37^{\circ} \mathrm{C}$. The tissue was washed with PBS. The slides with IL- $1 \propto$ and IL- 6 antibodies were then incubated with FITC-conjugated goat antirabbit $\operatorname{IgG}\left(1 / 20\right.$ dilution) for $30 \mathrm{~min}$ at $37^{\circ} \mathrm{C}$ and washed again with PBS. All slides were then mounted with $0.1 \% p$ phenylenediamine in PBS-glycerol and coded before examination. Treated sections were examined with UV light in an incident-light fluorescent microscope, and the fluorescence pattern (focal, diffuse; mesangial, loop) was defined. Fluorescence intensity was graded as negative, or $1+$ through $4+$. Control sections lacking primary antibody were included with each experiment. All sections were examined by two authors (T.R.W. and D.W.) independently.

Histology. Paraffin-fixed tissue was cut into 4- to $6-\mu \mathrm{m}$ sections and stained with H\&E, PAS, and Jones silver- methenamine by standard methods. Sections were coded and examined independently by two of the authors (T.R.W. and D.W.) using a variation of the method we have described previously (6). Glomerular and interstitial regions were each scored. Glomeruli were scored on a scale of a 0 (absent) to $4+$ (extensive, involving all glomeruli) for mesangial proliferation and mesangial matrix increase. The interstitium was similarly scored for peritubular inflammation, peritubular matrix increase, and tubular atrophy.

In situ hybridization. Paraformaldehyde-fixed tissue was used for C3 in situ hybridization, as previously described from our laboratory (3-5). Sense strand riboprobes were included in all experiments as negative controls, and mouse liver was used as a positive tissue control.

Sections were examined by dark-field microscopy at $\times 20$ and $\times 40$. The presence, distribution, and intensity of signal was recorded for each tissue by two of the authors (T.R.W. and D.W.). Signal that was not different from sense strand control (such as Fig. $1 F$ ) was graded as 0 , whereas occasional scattered foci were graded $1+$. Focal tubular label involving $\leq 50 \%$ of control tubules (such as Fig. $1 G$ ) was graded 2+. Label involving $>50 \%$ of tubules was $3+$, whereas $>75 \%$ (Fig. $1 H$ ) constituted a $4+$ label. A similar scale was applied to periglomerular (Bowman's capsule) label.

Experimental protocol. Animals received injections of HSA (4 mg) intraperitoneally five times weekly, with LPS $(0.05 \mathrm{mg})$ intraperitoneally three times weekly. Control animals received $0.15 \mathrm{M} \mathrm{NaCl}(0.1 \mathrm{~mL})$ five times weekly. At intervals of $1 \mathrm{wk}$ for a total of $6 \mathrm{wk}$, one saline and three HSA/LPS-treated animals were killed, with blood, urine, and tissue samples obtained as described.

This model was adapted from a previously described method (7). In pilot studies undertaken to determine the optimal immunization schedule, we determined that extending the HSA immunization from the original $14 \mathrm{~d}$ and adding LPS as an immune adjuvant produced the most consistent, reproducible lesion.

\section{RESULTS}

\section{Histology}

Control animals. The appearance of the saline-injected control animals was uniform at each weekly interval. No changes in mesangial cellularity or matrix or in the morphology of the tubulointerstitial areas were seen (Fig. 1A).

Treated animals. Between wk 1 and 3, there was a uniform, progressive increase in mesangial matrix and cellularity. No interstitial lesions were present. At $4 \mathrm{wk}$, the glomerular lesions were more advanced, and rare foci of periglomerular and peritubular mononuclear infiltration were present (Fig. $1 B)$. At wk 5, the glomerular changes included some obliteration, which involved half the glomeruli by wk 6 . The tubular changes also progressed, with widespread infiltrate and occasional atrophy at wk 5, followed by extensive fibrosis, and atrophy at wk 6 (Fig. 1C). Most sections at wk 6 showed essentially end-stage renal disease. 

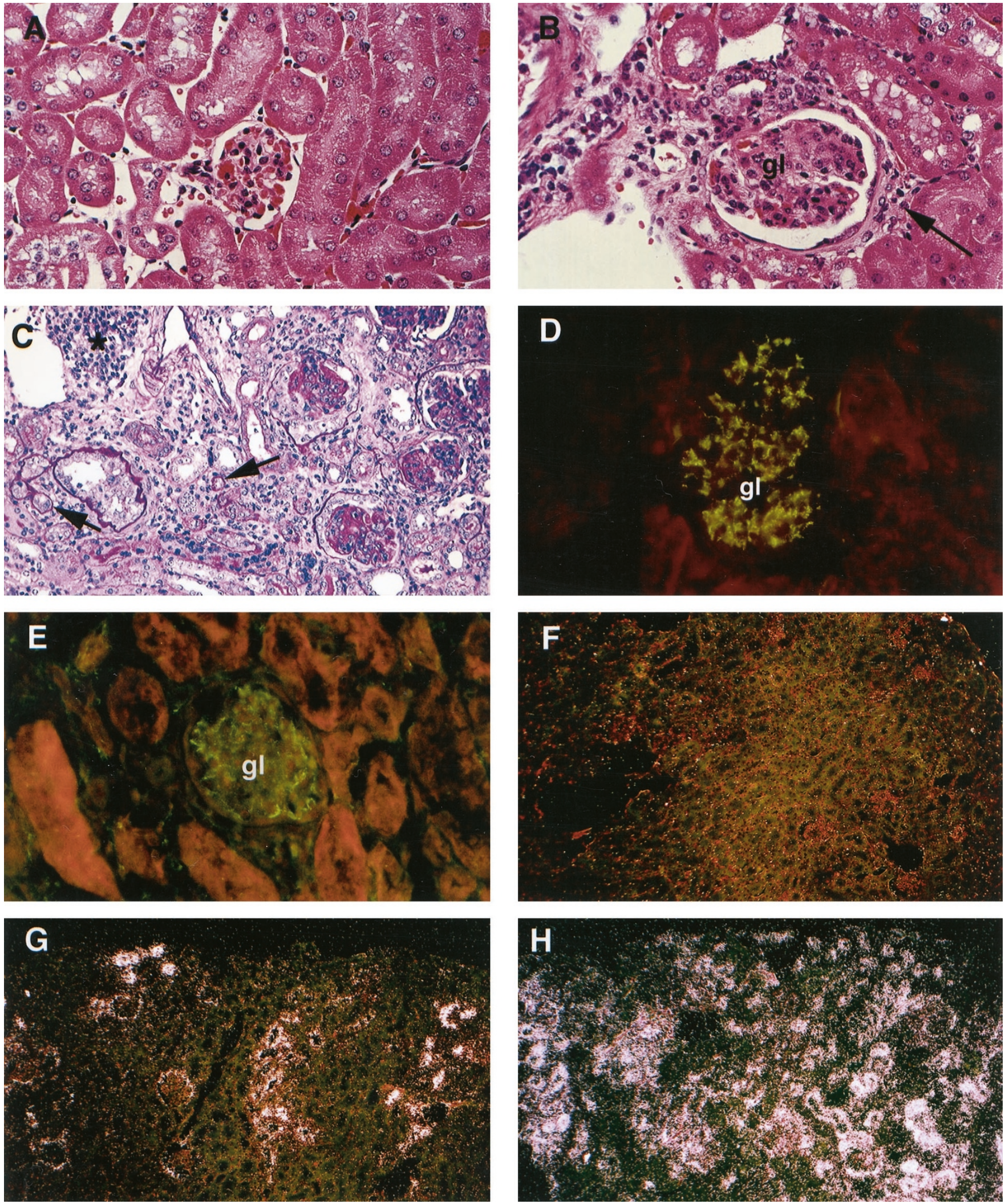

Figure 1. $A, H \& E$-stained section from saline-injected animal at 4 wk to illustrate normal glomerulus and interstitium $(\times 450)$; $B$, H\&E-stained section from HSA/LPS-injected animal at $4 \mathrm{wk}$, illustrating proliferative glomerulonephritis $(g l)$ and early periglomerular and peritubular infiltrate $($ arrow $)(\times 450)$; $C$, PAS-stained section from HSA/LPS-treated animal at $6 \mathrm{wk}$, illustrating extensive, advanced interstitial involvement $(\times 450) ; D$, $\propto \mathrm{C} 3 \mathrm{c}$-stained section from HSA/LPS-treated animal at $2 \mathrm{wk}$, illustrating early presence of complement deposits in glomerular mesangium $(g l)(\times 500) ; E$, IL-1 $\propto$-stained section from HSA/LPS-treated animal at $4 \mathrm{wk}$, illustrating presence of cytokine in a mesangial distribution $(g l)(\times 500) ; F$, C3 in situ hybridization of section from HSA/LPS-treated animal at $1 \mathrm{wk}$, illustrating absence of signal early $(\times 100) ; G$, C3 in situ hybridization of section from HSA/LPS-treated animal at 4 wk illustrating focal signal in a tubular and periglomerular distribution $(\times 100), H, \mathrm{C} 3$ in situ hybridization of section from HSA/LPS-treated animal at 5 wk, illustrating extensive, generalized tubular and periglomerular signal $(\times 100)$. 


\section{Immunohistochemistry}

At wk 1, there was no fluorescence with any antisera. Coarse granular deposits $(1+)$ of $\mathrm{C} 3 \mathrm{c}$ first appeared at wk 2 (Fig. 1D), and progressed to a generalized mesangial pattern that was well established $(2+)$ by wk 3 and continued through wk $6(3+)$. Beginning at wk 4, some tubular $\mathrm{C} 3 \mathrm{c}$ protein was also present.

The IL- $1 \propto$ and IL- 6 patterns were similar. Generalized trace reaction for both cytokines in a mesangial distribution $(1+)$ was first detected at $2 \mathrm{wk}$. This was well-established by 3-4 wk $(2+)$, and continued unchanged through the end of the study (Fig. 1E).

\section{C3 mRNA by in situ hybridization}

Control hybridization of the sense and antisense $\mathrm{C} 3$ riboprobe on murine liver confirmed its activity and specificity. No C3 mRNA was seen in the kidney tissue of saline-injected mice at any time.

The treated animals showed no C 3 mRNA on wk 1 or 2 (Fig. $1 F)$. By wk 3, however, definite foci of signal were detected in a periglomerular and proximal tubular location $(1+)$. By $4 \mathrm{wk}$, this activity was more extensive $(2+)$, and the signal was stronger (Fig. 1G). Progressive increase in signal intensity and distribution occurred on wk $5(3+)$ and $6(4+)$ (Fig. $1 H)$.

With the exception of the periglomerular signal (from the epithelium of Bowman's capsule), there was no evidence of glomerular C3 mRNA at any time. This observation was maintained even in situations in which the histology demonstrated an extensive glomerular infiltrate and immunohistochemistry showed extensive C3 protein deposits.

Also, the interstitial signal was restricted to proximal tubular epithelial cells. At no time was there evidence of message in infiltrating inflammatory cells or in blood vessels. Lowmagnification scanning of sections never showed signal in the medullary portions of the kidney.

\section{Clinical disease}

The animals had hematuria, proteinuria, and progressive renal insufficiency (elevated urea nitrogen), as detailed in Fig. 2. Two treated animals died, one each at 2 and $3 \mathrm{wk}$.

\section{Integration of findings}

In Figure 2, the sequence of evolution of these findings is summarized. The depth of shading in this figure represents the $0-4+$ scales described in "Methods." It is notable that glomerular cytokine activity is established by wk 2 , before any $\mathrm{C} 3$ mRNA is seen. In turn, development of interstitial C3 message at 3-4 wk clearly antedates the development of tubular injury, which is rare and spotty at wk 4 and not established until wk 5. Finally, the most dramatic change in clinical findings (urinalysis and blood urea nitrogen) do not begin until tubulointerstitial injury is established.

\section{DISCUSSION}

Most reported models of glomerulonephritis concentrate on the development and evolution of the glomerular lesion. In human disease, however, the severity of glomerulonephritis is nearly always marked by the nature and extent of the tubulointerstitial involvement. In systemic lupus erythematosus, for example, Esdaile et al. (8) have shown that the factor most predictive of deterioration in renal function is the presence of interstitial involvement in the initial kidney biopsy. Similar observations have been made in $\operatorname{IgA}$ nephropathy $(9,10)$ and

SUMMARY OF FINDINGS IN HSA/LPS TREATED MICE

Mesangial proliferation
Mesangial matrix increase
Mesangial C3C
Mesangial IL-1
Mesangial IL-6
Tubular C3 mRNA
Peri-glomerular C3 mRNA
Tubular C3C
Peri-tubular inflammation
Peri-tubular matrix increase
Tubular atrophy
Urine Protein/Blood
Blood urea nitrogen mg/dl (SD)
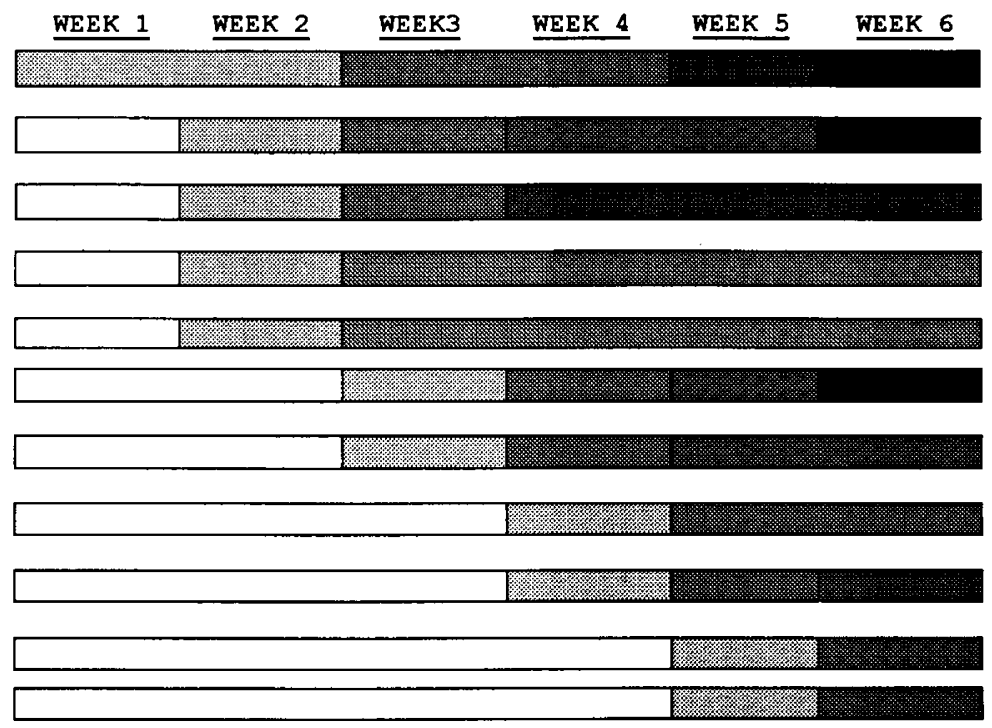

$\begin{array}{llllll}\mathrm{tr} / \mathrm{tr} & 1+/ \mathrm{tr} & 2+/ \mathrm{tr} & 1+/ \mathrm{sm} & 2+/ \bmod & 2+/ 1 \mathrm{~g} \\ 21(6) & 32(6) & 24(4) & 37(11) & 45(31) & 50(45)\end{array}$

Figure 2. Summary of clinical, histologic, immunohistochemical, and in situ hybridization findings in treated animals. The degree of shading in the bars represents the 0 to $4+$ scale used in tissue analysis (see text for details). The scales for urine blood (trace to large) and protein (trace to $4+$ ) are based on the dipstick method used. 
membranoproliferative glomerulonephritis (11). Tubulointerstitial injury per se results in deterioration in renal function $(1$, 12).

The mechanism by which some glomerular inflammatory processes lead to tubulointerstitial disease are not well understood. Such an understanding, however, is potentially very important. First, it might allow the early recognition of disorders at high risk of progression. Second, it might permit the design of new treatment strategies.

With human biopsy tissue studies, we have suggested a role for tubular complement synthesis in this process. Although complement component $\mathrm{C} 4$ appears to be expressed constitutively in all human kidneys studied (3), C3 (4) and B (5) are differentially regulated.

By in situ hybridization, message for these two components of the alternative pathway $\mathrm{C} 3$ convertase are expressed in the proximal tubular epithelium of kidneys with interstitial disease. In the case of $\mathrm{C} 3$, this observation has been confirmed by others (13). Although one group has reported C3 expression by glomerular cells as well (14), the equal intensity of tubular message in this study, in our opinion, makes the observation questionable. Even if the low-level glomerular expression in this report were real, tubular expression should have been proportionately much more extensive. In any case, it is difficult to ascribe importance to low levels of locally produced complement in a structure (the glomerulus) continuously bathed with plasma components.

Evidence of human renal C3 synthesis has led many to suggest a role for local complement in disease. For the most part, however, these suggestions have related to a role in glomerular inflammation (15). Our work, however, has led to the generation of a hypothesis that relates local complement synthesis to progressive interstitial injury. This hypothesis is based both on our work and on that of others, and is the basis for the studies described here. According to this hypothesis, the initial insult in most immune glomerulonephritides includes direct complement-mediated glomerular injury. The complement source for this initial step is the circulation (16).

In the course of this glomerular injury, a variety of cytokines are expressed in the mesangium, either synthesized by resident mesangial cells or by infiltrating inflammatory cells $(17,18)$. Several of these cytokines (IL-1 and IL-6, for example), have been shown to up-regulate C3 transcription (19). We suggest that these cytokines may gain access to the proximal tubular epithelium and the epithelium of Bowman's capsule via glomerular filtrate or, in the case of the proximal tubule, the vasa recta. This, in turn, could up-regulate $\mathrm{C} 3$ expression in these cells.

Once C3 protein is present in the interstitium, there are a variety of ways in which it could be activated. Local cell injury, for example, could result in the basement membrane of some cells becoming alternative pathway activators. In one in vitro study (20), proximal tubular epithelial cells in culture were shown to be alternative pathway activators. The ammonia content of the interstitium $(21,22)$ may also result in the formation of $\mathrm{NH}_{3} \cdot \mathrm{C} 3$, an effective convertase.

In any event, local production and activation of $\mathrm{C} 3$ would then be capable of mediating interstitial effects in a variety of ways. Nonlytic insertion of the MAC into the cell could result in expression of a variety of proteins, which, in turn, could mediate progressive inflammation, fibrosis, or cell death (23). Occupation of C5a receptors could have similar results. Fluid-phase anaphylatoxins could also be generated by this process, further recruiting inflammatory cells to the peritubular environment.

Both our human biopsy studies (3-5) and the model described herein are consistent with this hypothesis. These data suggest that the role of complement in glomerulonephritis may include, in addition to the well-established induction of glomerular injury, mediating the chronic interstitial injury that accompanies progressive disease. The availability of this wellcharacterized model will now permit a more precise study of the role of complement with gene targeting, as well as analysis of genes induced in tubular cells by complement deposition. Such studies, currently ongoing, should also make it possible to define the complement dependence of the model.

Acknowledgments. The authors thank Allene Ford and Jan Messer for careful secretarial assistance, and Alicia Emley for skilled technical assistance.

\section{REFERENCES}

1. Mackensen-Haen S, Bohle A, Christensen J, Wehrmann M, Kendziorra H, Fokot F 1992 The consequences for renal function of widening of the interstitium and changes in the tubular epithelium of the renal cortex and outer medulla in various renal diseases. Clin Nephrol 37:70-77

2. Bohle A, Mackensen-Haen S, von Gise H, Grund KE, Wehrmann M, Batz C, Bogenschultz O, Schmitt H, Nagy J, Muller C 1990 The consequences of tubulointerstitial changes for renal function in glomerulonephritis. Pathol Res Pract 186:135-144

3. Witte DP, Welch TR, Beischel LS 1991 Detection and cellular localization of human $\mathrm{C} 4$ gene expression in the renal tubular epithelial cells and other extrahepatic epithelial sources. Am J Pathol 139:717-724

4. Welch TR, Beischel LS, Witte DP 1993 Differential expression of complement C3 and C4 in the human kidney. J Clin Invest 92:1451-1458

5. Welch TR, Beischel LS, Frenzke M, Witte D 1996 Regulated expression of complement factor B in the human kidney. Kidney Int 50:521-525

6. Welch TR, Fryer C, Shely E, Witte DP, Quinlan M 1992 Double-blind, controlled trial of short-term prednisone therapy in immunoglobulin A glomerulonephritis. J Pediatr 121:474-477

7. Iskandar SS, Gifford DR, Emancipator SN 1988 Immune complex acute necrotizing glomerulonephritis with progression to diffuse glomerulosclerosis Lab Invest 59:772779

8. Esdaile JM, Levinton C, Federgreen W, Hayslett JP, Kashgarian MK 1989 The clinical and renal biopsy predictors of long-term outcome in lupus nephritis: a study of 87 patients and review of the literature. Q J Med 269:779-833

9. Bogenschutz O, Bohle A, Batz C, Wehrmann M, Pressler H, Kendziorra H, Gartner HV 1990 IgA nephritis: in the importance of morphological and clinical parameters in the long-term prognosis of 239 patients. Am J Nephrol 10:137-147

10. Lee S-MK, Rao VM, Franklin WA, Schiffer MS, Aronson AJ, Spargo BH, Katz AI 1982 IgA nephropathy: morphologic predictors of progressive renal disease. Hum Pathol 13:314-322

11. Schmitt H, Bohle A, Reineke T, Mayer-Eichberger D, Wolfgang V 1990 Long-term prognosis of membranoproliferative glomerulonephritis type I. Nephron 55:242-250

12. Bohle A, Mackensen-Haen S, von Gise H 1987 Significance of tubulointerstitial changes in the renal cortex for the excretory function and concentration ability of the kidney: a morphometric contribution. Am J Nephrol 7:421-433

13. Montinaro V, Gesualdo L, Ranieri E, Monno R, Grandaliano G, Schena FP 1997 Renal cortical complement C3 gene expression in IgA nephropathy. J Am Soc Nephrol 8:415-425

14. Miyazaki M, Abe K, Koji T, Furusu A, Ozono Y, Harada T, Nakane PK, Yagame M, Endoh M, Nomoto Y 1996 Intraglomerular C3 synthesis in human kidney detected by in situ hybridization. J Am Soc Nephrol 7:2428-2433

15. Sacks SH, Zhou W, Sheerin NS 1996 Complement synthesis in the injured kidney: does it have a role in immune complex glomerulonephritis? J Am Soc Nephrol 7:2314-2319

16. Hebert LA, Fernando GC, Birmingham DJ 1992 The role of the complement system in renal injury. Semin Nephrol 12:408-427

17. Chen W-P, Lin C-Y 1994 Augmented expression of interleukin-6 and interleukin-1 genes in the mesangium of IgM mesangial nephropathy. Nephron 68:10-19

18. Horii Y, Muraguchi A, Iwano M, Matsuda T, Hirayama T, Yamada H, Fujii Y, Dohi K, Ishikawa H, Ohmoto Y, Yoshizaki K, Hirano T, Kishimoto T 1989 Involvement of IL-6 in mesangial proliferative glomerulonephritis. J Immunol $143: 3949-3955$ 
19. Falus A, Rokita H, Walcz E, Brozik M, Hidvegi T, Meretey K 1990 Hormonal regulation of complement bio-synthesis in human cell lines. II: upregulation of the biosynthesis of complement components $\mathrm{C} 3$, factor $\mathrm{B}$ and $\mathrm{C} 1$ inhibitor by interleukin-6 and interleukin-1 in human hepatoma cell line. Mol Immunol 27:197-201

20. Biancone L, David S, Pietra VD, Montrucchio G, Cambi V, Camussi G 1994 Alternative pathway activation of complement by cultured human proximal tubular epithelial cells. Kidney Int 45:451-460
21. Gordon DL, Krueger RA, Quie PG, Hostetter MK 1985 Amidation of C3 at the thiolester site: stimulation of chemiluminescence and phagocytosis by a new inflammatory mediator. J Immunol 134:3339-3345

22. Nath KA, Hostetter MK, Hostetter TH 1989 Ammonia complement interaction in the pathogenesis of progressive renal injury. Kidney Int 36:52-54

23. Morgan BP 1989 Complement membrane attack on nucleated cells: resistance, recovery and non-lethal effects. Biochem J 264:1-14 